RETRACTION

doi:10.1038/nature12897

\title{
Retraction: The NAD-dependent deacetylase SIRT2 is required for programmed necrosis
}

Nisha Narayan, In Hye Lee, Ronen Borenstein, Junhui Sun, Renee Wong, Guang Tong, Maria M. Fergusson, Jie Liu, Ilsa I. Rovira, Hwei-Ling Cheng, Guanghui Wang, Marjan Gucek, David Lombard, Fredrick W. Alt, Michael N. Sack, Elizabeth Murphy, Liu Cao \& Toren Finkel

Nature 492, 199-204 (2012); doi:10.1038/nature11700

We retract this Article because some of the data, specifically the data reported in Fig. 2 demonstrating an in vitro requirement for Sirt 2 in TNF- $\alpha$-mediated necroptosis, appears to be irreproducible. We and others have confirmed that Sirt2 and RIP3 interact, and we continue to believe that the absence of Sirt 2 protects against ischaemic myocardial damage. Nonetheless, our inability to reproduce the data in Fig. 2 involving TNF- $\alpha$-mediated necroptosis undermines our confidence in the scientific conclusions reported and the proposed mechanism. Although the matter is currently under further review, we wish to retract the Article in its entirety, and regret any adverse consequences that may have resulted from the paper's publication. 